\title{
Multi-Hop Effective Bandwidth Based Routing in Multi-Radio Wireless Mesh Networks
}

\author{
Hongkun Li, Yu Cheng, Chi Zhou \\ Department of Electrical and Computer Engineering, \\ Illinois Institute of Technology, Chicago, IL, USA \\ Emails: $\{$ hli55, cheng, zhou $\} @$ iit.edu
}

\begin{abstract}
In this paper, we address the routing issue in a wireless mesh network, where each node is stationary and equipped with one or more radios. Specifically, we propose a new path metric called multi-hop effective bandwidth (MHEB), which provides a generic approach to calculate the achievable bandwidth along a path, taking the impacts of inter/intra-flow interference and space/channel diversity into account. We also present a new routing protocol based on the MHEB metric, which achieves interference-aware and load-balancing routing at the same time. Performance of the MHEB based routing in improving the network throughput is validated through computer simulations, compared with the existing popular routing metrics.
\end{abstract}

\section{INTRODUCTION}

In recent years, wireless mesh networks (WMNs) [1] have attracted wide attention due to their desirable features, including self-organization, self-configuration, easy maintenance, reliable service coverage, and broadband access especially when multiple channels or radio interfaces are applied. In a multi-hop WMN, it is always preferable to choose a path with higher throughput between a pair of source/destination nodes to fully exploit the network capacity [7].

In a multi-radio multi-channel wireless mesh network, each channel may observe different traffic loads and different interference topologies, where how to properly design the routing metric and protocol to fully exploit the space and channel diversity is much challenging than that in a single-channel context [4]. In this paper, we consider that the medium access control (MAC) protocol applied over each channel is carrier sensing multiple access with collision avoidance (CSMA/CA) based, e.g. IEEE 802.11. Each multi-hop flow may encounter channel contentions not only from other flows passing around, but also from the flow itself where upstream and downstream hops close enough contend the channel. Systematically, the interference in a multi-hop wireless network can be classified into two types: inter-flow and intra-flow [12]. To achieve higher end-to-end throughput, the routing protocol is expected to select a path experiencing less interference. How to effectively incorporate the impacts of inter/intra- flow interferences into the routing metric is a hot open research issue.

The routing metric plays an extremely critical role in a routing protocol. The studies in [2], [6], [8] design routing metrics for load balancing routing in a multi-hop wireless network. To exploit the space diversity, the link conflict graph is normally applied to model the interference among different hops in [9], [10], and the interference clique transmission time is proposed as a routing metric in [10]. However, the conflict graph based approaches normally induce large computation overhead in searching for the maximal independent sets or cliques, and are not suitable for dynamic distributed routing protocols. De Couto et al. propose the metric of expected transmission count (ETX) [11] to describe the channel contention level experienced by a wireless link, which works well in a homogeneous single-radio environment. However, ETX is not capable of describing the complex scenarios in a multiradio wireless mesh network, normally involving inter/intraflow interferences and different rate/interference/topology profiles over different channels. The link metric of expected transmission time (ETT) and the associated path metric of weighted cumulative ETT (WCETT) are proposed in [4] for multi-radio mesh networks, which enhance the ETX to count the heterogeneous channel rate and intra-flow interference, but the inter-flow interference and the space diversity are still not considered. The metric of interference and channel switching (MIC) [5] incorporates both inter-flow and intraflow interference, whereas it is not accurate enough to capture the impact of interference magnitude on path capacity.

In this paper, we propose a new path metric called multihop effective bandwidth (MHEB), which provides a generic approach to accurately capture the impacts of both inter/intraflow interference and space/channel diversity along a path. We consider a practical scenario that an end-to-end path may consist of both multi-radio hops and single-radio hops, where different channels do not interfere with each other but interference exists in the same channel. We particularly develop a sub-path based iterative approach to compute the achievable bandwidth under intra-flow interference (ABIRF) as well as exploit the channel and space diversity. To accurately capture the inter-flow interference, we combine the ETX with a newly proposed interference degree ratio to evaluate the achievable bandwidth under the inter-flow interference (ABITF). The path metric MHEB is defined as the weighted average of ABIRF and ABITF. With computer simulations and comparison with the hop-count based routing and WCETT based routing, we demonstrate that MHEB based routing can obviously improve the throughput in a multi-radio wireless mesh network.

The reminder of this paper is organized as follows. Section 
II describes the system model. Section III derives the path metric of MHEB, and presents the new routing protocol based on MHEB. Simulation results and performance evaluation are given in IV. Section V concludes this study.

\section{SYSTEM MODEL}

We consider a wireless mesh network, where each node may have one or more network interface cards (NICs) and the number of NICs equipped at different nodes is not necessarily the same. For a multi-radio node, different NICs are assumed to work on separate channels without inter-channel interference. All the nodes are stationary, and any one can be used as a router. The wireless links between nodes are bidirectional. We define the transmission range of a node as one hop, while the interference range is $r(\geq 2)$ hops. We consider that the WMN operates over the IEEE 802.11 based MAC, and assume that the routing control information exchanged among neighbor nodes is error free. We further assume that the channel assignment at each node is given. Note that the channel assignment issue in a multi-radio WMN itself is a very challenging issue [13], and is out of the scope of this paper.

We adopt the well-known physical model presented in [9] to describe the interference among different hops. Such an interference model indicates that a transmission from node $u$ to $v$ is successful if the signal to interference and noise ratio (SINR) at receiver $v$ is above the pre-determined threshold $\gamma$, i.e.,

$$
\frac{P_{v}(u)}{N+\sum_{k \in v^{\prime}} P_{v}(k)} \geq \gamma
$$

where $N$ denotes the background noise, $P_{v}(u)$ the received power at node $v$ from node $u, v$ t the set of nodes located in interference range of $v$, and $P_{v}(k)$ the interference power from a interfering node $k$. For convenience, we use the acronyms ITFI and IRFI to represent the inter-flow interference and intraflow interference, respectively.

\section{Design of Interference Aware Routing Metric}

In this section, we will discuss how to accurately quantify inter-flow and intra-flow interference along a path. In particular, we are to derive available bandwidth of a path under interferences, which is termed as multi-hop effective bandwidth (MHEB), and will be used as the path metric.

To show necessity and motivation of our metric, we would like to analyze two popular metrics in interference-aware routing. WCETT considers IRFI but overlooks the ITFI. Even for IRFI, WCETT involves all the links without considering the space diversity that links far from each other can transmit simultaneously. In Fig.1, $S R$ is the router with single radio while $M R$ is the one with multiple radios. According to the algorithm in [4], the path cost for two paths are $0.5 \times(6 E T T 1+E T T 2)$ and $0.5 \times(6 E T T 1)$, with $\beta=0.5$. It is natural to choose path 2 due to its smaller metric value. However, if the links (SR1,MR1) and (SR2,SR5) could transmit simultaneously, the IRFI of path 1 should be $2 E T T 1$

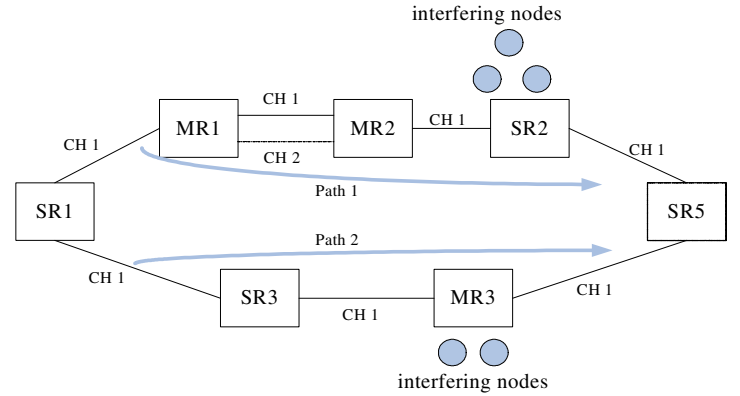

Fig. 1. Illustration of path selection based on WCETT and MIC.

instead of $3 E T T 1$. Consequently we need a mechanism to identify which sets of links should be contained when calculating IRFI. For MIC [5], it only considers the number of interfering nodes, while failing to consider the magnitude of the interference. MIC prefers path 2 because it has lower value for ITFI. Nevertheless, if the two interfering neighbors of MR3 are heavily loaded, resulting in large interfering power, while MR2's neighbors have light traffic, the choice becomes unreasonable any more. Another limitation arises from the fact that there is no clear physical meaning for the value of $w_{1}$ and $w_{2}$ when estimating the IRFI. As a result, we need to find a proper way to reflect the magnitude of interference and a suitable measurement function for IRFI.

\section{A. Calculation of Multi-hop Effective Bandwidth}

To measure the impact on the capacity, we can get some useful information by focusing on the received power. With our interference model, the SINR threshold implicitly denotes the maximum interference a node could tolerate to process a successful communication. We define interference degree ratio $I D R_{i}(u v)$ for link $i$ between $u$ and $v$ as follows:

$$
I D R_{i}(u v)=\frac{\sum_{k \in v^{\prime}} P_{v}(k)}{P_{\max }}
$$

The ratio reflects the utilization of the channel assigned to link $i . P_{\max }$ is the maximum tolerable interference power at receiver and can be calculated by (1). $\sum_{k \in v} P_{v}(k)$ is the sum of all undesired power from other transmissions at node $v$. Note that if there is no interference, the ratio is 0 , implying that entire bandwidth of this channel is available for link $i$. If the ratio is 1 , indicating that the channel has been fully occupied by other links, this also means no additional bandwidth can be allocated for link $i$ until the ratio becomes less than 1. Based on this definition, we evaluate the achievable bandwidth at link $i$ under the ITFI as follows:

$$
A_{B I T F_{i}}=\left(1-I D R_{i}\right) * B_{i} / E T X_{i}
$$

where, $B_{i}$ is the original bandwidth of link $i$;ETX $X_{i}$ [11] denotes the expected transmission attempts for a successful transmission over link $i$. It reflects the channel quality of link $i$. $\left(1-I D R_{i}\right) * B_{i}$ indicates ITFI level around link $i$. The physical meaning of (3) is that: given the packet loss rate $p$, a successful transmission needs $E T X_{i}$ attempts in average, so 


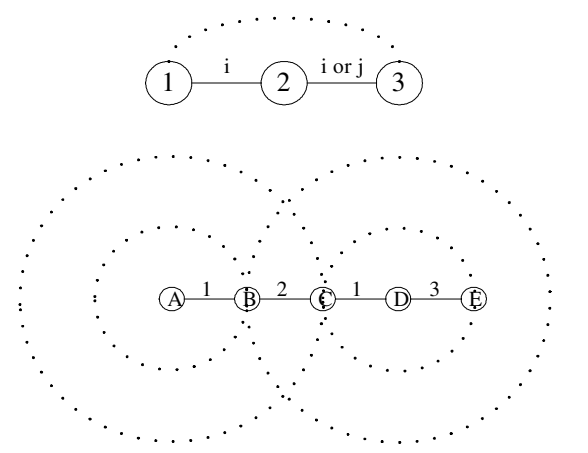

Fig. 2. Computation of the achievable bandwidth under intra-flow interference (ABIRF).

during these $E T X_{i}$ transmissions, the bandwidth is effectively used for only one transmission.

There exists IRFI if two links are in the same path and within each other's interference range, i.e. within $r(\geq 2)$ hops. Since we assume the transmission range is one hop, $r$ is also the ratio of interference range to transmission range. We define a new concept of sub-path based on the observation that given $r$, a link will potentially interfere with another link, which is at most $r+2$ hops away, and each sub-path spans $r+2$ links, which is illustrated in Fig.2. We define a sub-path containing $r+2$ consecutive links in a path, and suppose that there are $Q$ hops in a path, there would be $Q-r-1$ sub-paths in the path. Now, we are in the position to measure the effective bandwidth of a path. As depicted in Fig.2, if two successive links share one channel, say channel $i$, with its maximum bandwidth $B_{i}$, the achievable bandwidth for these two links is $B_{i} / 2$. Actually, we could consider the two physical links to be a virtual link with $B_{i} / 2$ effective bandwidth from node 1 to node 3 . In general, if two links are assigned the same channels, $i$ and $j$, and they send one packet in two different slots. We can compute the achievable bandwidth under the IRTF as follows:

$$
A B I R F=\frac{L}{\frac{L}{B_{i}}+\frac{L}{B_{j}}}=\frac{B_{i} \times B_{j}}{B_{i}+B_{j}}
$$

where $L$ is packet length. Note that this situation is possible within a sub-path when $B_{i}$ is effective bandwidth for the set of previous links and channel $j$ has been used in previous links. When two links work on different channels, they could send and receive packets simultaneously with different radios. The effective bandwidth for these two links is $\min \left(B_{i}, B_{j}\right)$. This result is easily understandable: during a long time period, the link with smaller bandwidth becomes the bottleneck for the two-link transmission. For general situation, we could calculate the effective capacity of a sub-path by following iterative way:

Step 1: For first link of $j$ th sub-path, set $A B I R F_{j}$ equals to bandwidth of the channel on which first link works.

Step 2: Set $B_{\text {pre }}=A B I R F_{j}$ and go to the next link, and check whether the channel $i$ on which next link is working has been used in previous links, if no, go to step 3; otherwise go to step 4
Step 3: In this case, set

$$
A B I R F_{j}=\min \left(B_{i}, B_{\text {pre }}\right)
$$

then go to step 5

Step 4: In this case, set

$$
A B I R F_{j}=\frac{B_{\text {pre }} \times B_{i}}{B_{\text {pre }}+B_{i}}
$$

then go to step 5

Step 5: check whether there is link still unconsidered in this sub-path, if yes, go to step 2; otherwise, terminate the iteration.

In the algorithm, $B_{\text {pre }}$ represents the equivalent ABIRF for previous links, $B_{i}$ is still the original bandwidth of link $i$. In Fig.2, consider the sub-path $A-B-C-D-E$. If $r=2$, then link $A B$ will potentially interfere with $D E$. We can use either (5) or (6) for each link. Initially, we set ABIRF equal to $B_{1}$. Then we go to link $B C$, Because $B C$ works on channel 2, link $B C$ and $A B$ could be active simultaneously. The corresponding bandwidth is $\min \left(B_{1}, B_{2}\right)$. For link $C D$, since channel 1 has already been used in previous two hops, the effective bandwidth from $A$ to $D$ is $\frac{B_{p r e} \times B_{1}}{B_{p r e}+B_{1}}$, here, $B_{\text {pre }}$ is bandwidth of virtual connection $A C$. Follow this step to the last link in this sub-path, we can get the ABIRF for this sub-path. Note that, our object is to find an accurate model to represent the IRFI, therefore we need to find the bandwidth for the whole path. We have divided a path into multiple subpaths. Based on this point, we define the IRFI of a path as follows:

$$
A B I R F=\min \left(A B I R F_{i}\right)
$$

where $i=1,2, \ldots Q-r-1$ is the sequence number of subpaths. If $Q-r-1 \leq 0$, we set $i=1$, which means the path is short and there is only one sub-path along the whole path. We pick the sub-path with least bandwidth as the IRFI of the whole path, because only one link could be active during one slot in a sub-path among the links on the same channel, and the sub-path with least effective bandwidth means it takes more time for the same flow to traverse these consecutive $r+2$ links than any other sub-paths. That results from more severe IRFI compared with other sub-paths. And therefore the new metric ABIRF is fully competent in reflecting the degree of IRFI of a path.

After introducing how to formulate two kinds of interference, we can define our new metric MHEB as follows:

$$
M H E B=\alpha \times \min \left(A B I T F_{i}\right)+(1-\alpha) \times A B I R F
$$

where $\alpha$ is a tunable parameter subject to $0 \leq \alpha \leq 1$. This setting could balance the contribution of two types of interference for the metric value. The first term denotes the bottleneck link over the whole path from ITFI point of view.

\section{B. Routing Protocol Design}

We also develop a new routing protocol to implement the MHEB based routing. Basically, our routing protocol can be characterized as multi-radio on-demand distance vector 
protocol, and can be achieved by modifying the popular AODV [3] protocol.

Each node needs to create an additional element, named MHEB, for each entry indicating a certain destination in its routing table. They also need to calculate the ABITF and ABIRF to update the value of MHEB when it receives an up-to-date RREQ or RREP. The HELLO packet is used to estimate ETX and IDR of each link. In our design, we assign one unique IP address for each interface of node, so that it is convenient to identify a node when it is working on different interfaces.

The RREQ message is uniquely identified by both source IP address and a broadcast id which is increased by 1 immediately when a RREQ packet is generated. Doing this could help prevent routing loops and redundant transmissions. To compute the path metric, each forwarding node inserts in RREQ information about IDR, ETX and the channels on which they are operating. Once an intermediate node receiving a RREQ, it first checks the IP address and broadcast id. In the case that it has already received this message, the node computes the ABIRF and ABITF based on the information in RREQ. If it is a new better path, the node will update the reverse route, attach the related information in RREQ, and forward it on each of its interface; otherwise, the node will discard the message. RREQ also contains a destination-only flag to denote whether an intermediate node is allowed to generate RREP packet.

The RREP message is unicast toward the source node along the reverse route recorded during transmission of RREQ message. During the propagation of RREP message, the intermediate nodes record the forwarding route to the destination node. When the source node receives the RREP message, it starts sending out the data packets through the pre-determined route. If the source could not receive a RREP for a period of time, it will resend RREQ until reaching the maximum number of retries.

When any intermediate node finds any link broken (by HELLO message), it sends out either a RRER packet or does a local repair by sending out a RREQ to find an alternative route to the destination node.

\section{Performance EVAluation}

\section{A. Simulation Model}

We evaluate the performance of the proposed routing metric and the new routing protocol by NS-2 simulation [14], [15]. We compare the throughput performance of MHEB with WCETT $(\beta=0.5)$ and Hop Count. We set two topologies for our simulation: the first is a grid topology shown in Fig.3, where 81 nodes are distributed within a $1800 \mathrm{~m} \times 1800 \mathrm{~m}$ area, and the area is divided into $200 m \times 200 m$ square cells. We place one node in each cell. We set 4 pairs of sources and destinations $\left(S_{i}, D_{i}\right)$ across the network, and change the per flow rate to study the performance. The other topology randomly generates 20 nodes in a $1500 \mathrm{~m} \times 1500 \mathrm{~m}$ area, and each node is placed with a random position and the topology keeps connectivity. For random topology, we setup two flows across the network. In each topology, the transmission range is

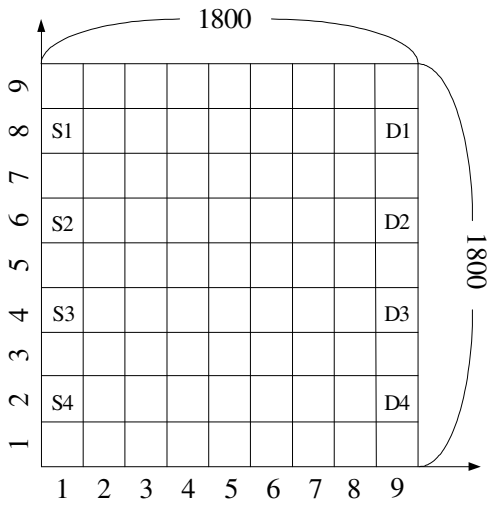

Fig. 3. The grid topology used in simulation.

$250 \mathrm{~m}$, while the interference range is $550 \mathrm{~m}$, so the ratio $r$ can be set to 2 . We run simulation under both single channel and multi-channel environments. Each flow is a CBR flow with packet size 1500 bytes. The running time is 100 seconds.

\section{B. Simulation Results}

Fig.4 and 5 show the network throughput when running the three metrics in the single channel environment. With single channel, the WCETT becomes ETT, and MHEB only includes ITFI. There is no need to consider the intra-flow interference under this scenario. As shown in Fig.4 and 5, MHEB has best performance in terms of throughput as expected, and hop count is the worst. Because MHEB considers the factor of inter-flow interference denoted by the IDR, while WCETT only captures the loss rate of the link and ignores the inter-flow interference. Even if there is only one channel, the MHEB outperforms WCETT. In addition, we can observe that all these three metrics have been saturated when the per flow rate increases to a certain level. This results from the exchange of control messages. Too much packet exchange leads to large overhead and precessing time in the system, consequently more packets are dropped, thus the throughput will not increase with the increment of flow rate any more.

Fig.6 and 7 illustrate the network throughput for different metrics under the multi-channel environment. Comparing with Fig.4 and 5, the throughput is much better even for the hop count. For multi-channel environment, the intra-flow interference is hardly negligible. In the grid topology, the source is normally more than 5 hops away from the destination for each flow. Since WCETT always considers all links in the computation of intra-flow interference even if they are out of others' interference ranges, which is inaccurate. MHEB intelligently divides a path into some sub-path and compute the ITFI and IRFI, that is the reason MHEB has the best performance.

\section{CONCLUSION}

In this paper, we concentrate on the routing problem in the 802.11-based multi-channel multi-radio wireless mesh network. All nodes are assumed fixed. We propose the new 


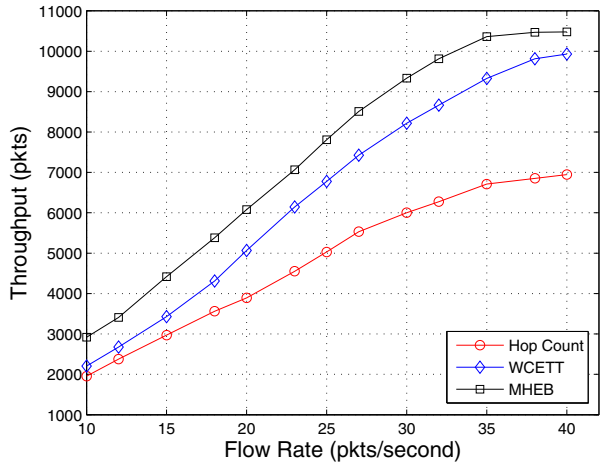

Fig. 4. Throughput performance with single channel in the grid topology.

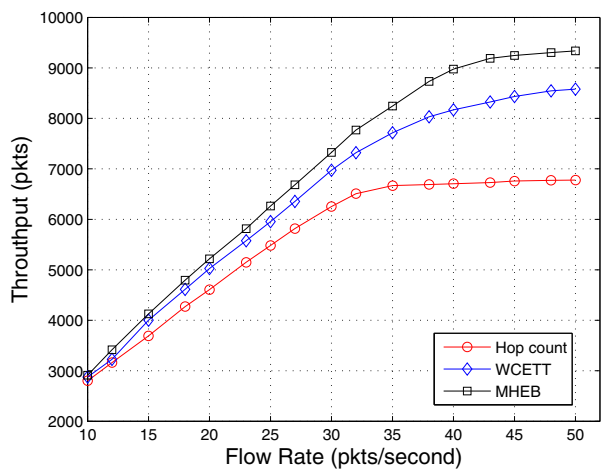

Fig. 5. Throughput performance with single channel in the random topology.

routing metric (MHEB) to reveal the impact of interference on the capacity of a path, which is measured by effective bandwidth. We also present a new on-demand routing protocol using this metric. The computation of MHEB considers both intra-flow and inter-flow interference, including factors such as link loss rate, channel utilization, and bandwidth of sub-path. The objective of MHEB is to choose the path experiencing the least interference from source to destination, resulting in better throughput performance. As a result, interferenceawareness and load-balancing can be achieved simultaneously. Simulation results show that out new metric outperforms the WCETT and hop count metrics in terms of the system throughput, because our proposed metric exploit the channel diversity and topology character more efficiently. However, our metric could not guarantee that we can always choose the optimal path. In our future work, we plan to develop algorithms to get the guaranteed optimal solution (optimal system throughput) by jointly designing channel assignment and routing problem.

\section{REFERENCES}

[1] I.F.Akyildiz, X. Wang, and W. Wang, "Wireless mesh networks: a survey," Computer Networks., 2005, pp.S23-S30.

[2] A. Raniwala, T.-c. Chiueh, "Architecture and Algorithms for an IEEE 802.11-Based Multi-Channel Wireless Mesh Network," in Proc. IEEE INFOCOM, 2005, pp.2223-2234.

[3] Charles Perkins, "Ad-hoc on-demand distance vector routing," in MILCOM panel on Ad Hoc Networks, 1997.

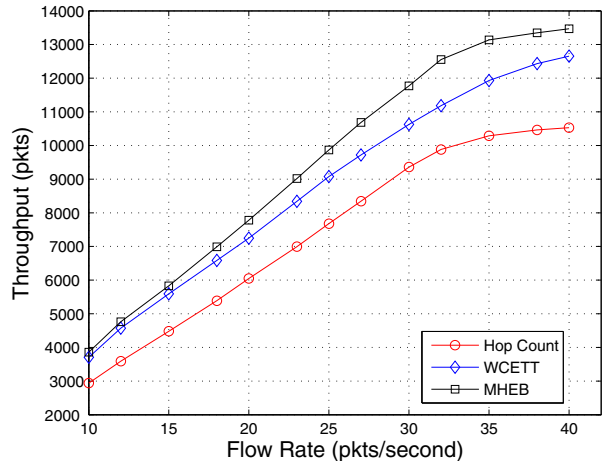

Fig. 6. Throughput performance with 3 channels in the grid topology.

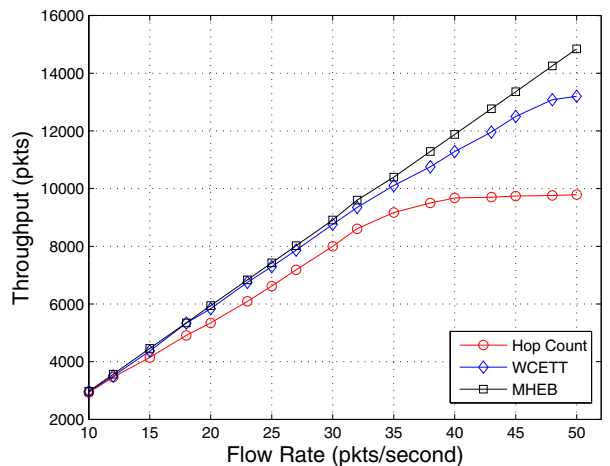

Fig. 7. Throughput performance with 3 channels in the random topology.

[4] Richard Draves, Jitendra Padhye, and Brian Zill, "Routing in MultiRadio, Multi-Hop Wireless Mesh Networks," in ACM MOBICOM, 2004, pp.114-128.

[5] Y. Yang, J. Wang, and R. Kravets, "Designing Routing Metrics for Mesh Networks," in WiMesh, 2005, .

[6] J.So, N. H.Vaidya, "Load-Balancing Routing in Multichannel Hybrid Wireless Networks With Single Network Interface," in IEEE Transactions on Vehicular Technology, 2007, Vol 56, pp.342-348.

[7] P. Kyasanur and N. H. Vaidya, "Routing and Interface Assignment in Multi-Channel Multi-Interface Wireless Networks," in Proc. IEEE WCNC, 2005, pp.2051-2056.

[8] Tehuang Liu, Wanjiun Liao, "Capacity-Aware Routing in Multi-Channel Multi-Rate Wireless Mesh Networks," in Proc. IEEE ICC, 2006, pp.1971-1976.

[9] K. Jain, J. Padhye, V. N. Padmanabhan, and L. Qiu, "Impact of Interference on Multi-hop Wireless Network Performance," in $A C M$ MOBICOM, 2003, pp.471-487.

[10] H. Zhai, Y. Fang "Impact of Routing Metrics on Path Capacity in Multirate and Multi-hop Wireless Ad Hoc Networks," in Proc. IEEE ICNP, 2007, pp.86-95.

[11] Douglas S. J. De Couto, Daniel Aguayo, John Bicket, and Robert Morris, "A High-Throughput Path Metric for Multi-Hop Wireless Routing," in ACM MOBICOM, 2003, pp.419-434.

[12] H. Zhai, J. Wang, Y. Fang, "Distributed packet scheduling for multihop flows in ad hoc networks," in Proc. IEEE WCNC, 2004, pp.1081-1086.

[13] K.N.Ramachandran, E.M.Belding, K.C.Almeroth, M.M.Buddhikot, "Interference-Aware Channel Assignment in Multi-Radio Wireless Mesh Networks," in Proc. IEEE INFOCOM, 2006, pp.1-12.

[14] Ramon Aguero Calvo, Jesus Perez Campo, "Adding Multiple Interface Support in NS-2," http://personales.unican.es/aguerocr/, 2007.

[15] The network simulator-ns2, http://www.isi.edu/nsnam/ns/.

[16] "Wireless Lan Medium Access Control (MAC) and Physical Layer (PHY) specifications," ANSI/IEEE Std 802.11: 1999 (E) Part 11, ISO/IEC 8802-11, 1999. 